\title{
ON ANALYTIC CONTINUATION FROM THE "EDGE OF THE WEDGE"
}

\author{
A. A. GONCHAR
}

The "edge of the wedge" theorem was first proved by N. N. Bogoliubov in 1956 in connection with applications to quantum field theory (see [7]). During the next years many various proofs, generalizations and refinements of Bogoliubov's theorem were obtained; the problems and results concerning this theorem constituted an important chapter in the theory of analytic functions of several complex variables and its applications (see [1-9]; an extensive bibliography can be found in V. S. Vladimirov's recent survey [9]).

In this note we present some "one-sided" versions of the "edge of the wedge" theorem, which are closely related with Malgrange-Zerner's theorem (see [3]). Our approach is based on the classical ideas and results of $\mathrm{R}$. Nevanlinna and $\mathrm{T}$. Carleman, connected with the notion of the harmonic measure.

\section{Notations}

Let $D_{j}$ be a Jordan domain in the complex $z_{j}$-plane, $E_{j}$ an open subset of the boundary $b D_{j}$ and $F_{j}=D_{j} \backslash E_{j}(j=1, \ldots, n) . D, E, F$ (the sets in $\left.C^{n}\right)$ are the direct products of $D_{j}, E_{j}, F_{j}, j=1, \ldots, n$, respectively.

$$
\begin{gathered}
V_{j}=E_{1} \times \ldots \times F_{j} \times \ldots \times E_{n}, \quad V_{j}^{\circ}=E_{1} \times \ldots \times D_{j} \times \ldots \times E_{n}=V_{j} \backslash E . \\
V=\bigcup_{j=1}^{n} V_{j}, \quad V^{\circ}=\bigcup_{j=1}^{n} V_{j}^{\circ}=V \backslash E .
\end{gathered}
$$

The harmonic measure of $b D_{j} \backslash E_{j}$, with respect to $D_{j}$ will be denoted by $h_{j} ; h_{j}$ is the generalized solution of the Dirichlet problem in $D_{j}$ for the boundary values 0 on $E_{j}$ and 1 on $b D_{j} \backslash E_{j}$. We define $h_{j}$ on $F_{j}$, putting $h_{j}=0$ on $E_{j}$; then $h_{j}$ is a function, continuous on $F_{j}$ and harmonic in $D_{j}$.

$$
h(z)=h\left(z_{1}, \ldots, z_{n}\right)=\sum_{j=1}^{n} h_{j}\left(z_{j}\right), \quad z \in F .
$$

$W=\{z \in F: h(z)<1\}, W^{\circ}=W \backslash E, H=$ int $W$ (the interior of $W$ in $C^{n}$ ).

The "edge" $E$ is an $n$-dimensional (real) subset of the boundary $b W(=b H)$. The $(n+1)$-dimensional subset $V^{\circ}$ of $b W$ consists of $n$ parts $V_{j}^{\circ}$; there is a single 
complex direction on each of these parts (the variable $z_{j}$ on $V_{j}^{\circ}$ ). All $V_{j}^{\circ}, j=1, \ldots, n$, are joined on their common border $E$, the edge of the "wedge" $W$.

On $e, C(e)$ denotes the class of continuous functions; $C R(e), e=V^{\circ}$ or $W^{\circ}$, denotes the class of continuous $C R$-functions on $e .|f|_{e}$ is the sup norm of $f$ on $e$.

\section{Theorems}

Theorem 1. Let $f \in C(V) \cap C R\left(V^{\circ}\right)$. Then there exists $\tilde{f} \in C(W) \cap C R\left(W^{\circ}\right)$ such that $\tilde{f}=f$ on $V$. If $|f|_{V}<+\infty$, then the following estimate holds:

$$
|\tilde{f}(z)| \leqq|f|_{E}^{1-h(z)}|f|_{V}^{h(z)}, \quad z \in W .
$$

The first statement of Theorem 1 means that if $f \in C(E)$ admits $C R$-continuation from the "edge" $E$ to $V$, then $f$ admits $C R$-continuation to the "wedge" $W$. We note that $\tilde{f} \in C(W) \cap C R\left(W^{\circ}\right)$ if and only if $\tilde{f}$ is continuous on $W$ and holomorphic in $H$. Clearly $H$ is the hull of holomorphy of the set $V(W$ is $C R$-hull of $V$ ); the characterization of the hull of holomorphy of $V$ in terms of the harmonic measures coincide with that of the "cross" in Siciak's theorem on separately analytic functions [6]. The estimate (1) is a version of the "two-constant theorem" for our geometrical configuration.

The harmonic measures $h_{j}$ are invariant under conformal mappings of the domains $D_{j}$; hence Theorem 1 is equivalent to the same assertion for any special choice of the domains $D_{j}, j=1, \ldots, n$. The following realizations are the most interesting:

$1^{\circ}$

$2^{\circ}$

$3^{\circ}$

$4^{\circ}$

$$
D_{j}=\left\{z_{j}: \operatorname{Im} z_{j}>0\right\}
$$

$$
D_{j}=\left\{z_{j}: 0<\operatorname{Im} z_{j}<b_{j}\right\}, \quad E_{j} \subset(-\infty,+\infty) ;
$$

$$
D_{j}=\left\{z_{j}:\left|\operatorname{Re} z_{j}\right|<a_{j}, 0<\operatorname{Im} z_{j}<b_{j}\right\}, \quad E_{j} \subset\left(-a_{j}, a_{j}\right) ;
$$

$$
D_{j}=\left\{z_{j}:\left|z_{j}\right|<1\right\}
$$

In these cases, $h_{j}, h$ and $W$ have a simple geometrical meaning. For instance, in the case $1^{\circ}, h_{j}=1-\alpha_{j} / \pi$, where $\alpha_{j}\left(z_{j}\right)$ is the angular measure of the set $E_{j}$ at the point $z_{j}$. If $E_{j}$ 's are (connected) arcs, it is convenient to realize $D_{j}$ 's as in $2^{\circ}$ or $3^{\circ}$ (putting $E_{j}=(-\infty,+\infty)$ and $E_{j}=\left(-a_{j}, a_{j}\right)$, respectively). In the case $2^{\circ}$ with $E_{j}=(-\infty,+\infty)$ we have: $h_{j}\left(z_{j}\right)=y_{j} / b_{j}, h(z)=y_{1} / b_{1}+\ldots+y_{n} / b_{n}$ and $W$ is the convex hull of $V$. One can obtain more general geometrical versions of Theorem 1 (in the cases $\left.1^{\circ}-3^{\circ}\right)$ after a linear transformation of the space $R_{y}^{n}\left(C_{z}^{n}=R_{x}^{n}+i R_{y}^{n}\right)$. 
Theorem 1 admits various generalizations. The statements about $C R$-continuation from $V^{\circ}$ to $W^{\circ}$ can be proved under weaker assumptions on the existence and coincidence of the boundary values of the functions $f_{j}=\left.f\right|_{V_{j}^{\circ}}$ on $E$. Moreover, the behaviour of $\tilde{f}$ for $z \rightarrow t^{\circ} \in E$ depends only on the local properties of the function $f(t), t \in E$, at the point $t^{\circ}$ (here $f$ is a common boundary value of the functions $f_{j}$ on $E)$. We will formulate the corresponding theorem for the bounded functions.

Suppose $E_{j}$ 's are rectifiable sets, $f \in C R\left(V^{\circ}\right)$ and $|f|_{V^{\circ}}<+\infty$; each function $f\left(\ldots, z_{j}, \ldots\right), z_{j} \in D_{j}$ (for any fixed variables $z_{k}=t_{k} \in E_{k}, k \neq j$ ) has nontangential boundary values for almost all $t_{j} \in E_{j}$. Hence the functions $f_{j}=\left.f\right|_{\nu_{j}^{\circ}}$ define the boundary functions $f_{j}(t), j=1, \ldots, n$, on the "edge" $E$. Under these assumptions and notations we have the following theorem.

Theorem 2. Let the boundary functions $f_{j}$ coincide almost everywhere on $E: f_{j}(t)=f(t)$ for almost all $t \in E$ with respect to the $n$-dimensional Lebesque measure. Then there exists $\tilde{f} \in C R\left(W^{\circ}\right)$ such that $\tilde{f}=f$ on $V^{\circ}$. If the function $f(t), t \in E$, is continuous at the point $t^{\circ} \in E$, then $\tilde{f}(z) \rightarrow f\left(t^{\circ}\right)$ for $z \rightarrow t^{\circ}, z \in W^{\circ}$.

The smoothness of $f(t), t \in E$, at the point $t^{\circ}$ implies almost the same smoothness of $\tilde{f}$ at this point. More precisely, if for some polynomial $T_{t^{\circ}}$ of degree $k$ we have $\left(f-T_{t^{\circ}}\right)(t)=O\left(\left|t-t^{\circ}\right|^{k+s}\right), 0<s<1$, for $t \rightarrow t^{\circ}, t \in E$, then $\left(f-T_{t^{\circ}}\right)(z)=O\left(\left|z-t^{\circ}\right|^{k+s^{\prime}}\right)$ for every $s^{\prime}<s$ and $z \rightarrow t^{\circ}, z \in W^{\circ}$.

\section{Method of the proof. Lemmas}

Without loss of generality we may assume that $E_{j}$ 's are rectifiable sets and $|f|_{E}<+\infty$. Let $\tilde{h}_{j}$ be a conjugate function for the harmonic measure $h_{j}\left(\tilde{h}_{j}\left(z_{j}^{\circ}\right)=0\right.$ for some fixed point $\left.z_{j}^{\circ} \in D_{j}\right), g_{j}=h_{j}+i \tilde{h}_{j}$ and

$$
g(z)=\sum_{j=1}^{n} g_{j}\left(z_{j}\right), \quad z \in D .
$$

We set

$$
K_{m}(f ; z)=\frac{1}{(2 \pi i)^{n}} \int_{E} e^{-m(g(t)-g(z))} \frac{f(t) d t}{\left(t_{1}-z_{1}\right) \ldots\left(t_{n}-z_{n}\right)}, \quad z \in D
$$

(each function $e^{-g_{j}}$ is bounded in $D_{j}$; we integrate in (2) nontangential boundary values of the corresponding functions).

If a function $f$ is holomorphic in $D$ and (say) continuous on $E$, then the following Carleman's formula holds:

$$
f(z)=\lim _{m \rightarrow \infty} K_{m}(f ; z), \quad z \in D
$$

(3) is easy to deduce from Cauchy's formula. 
The first (and the main) point of our approach is

L.emma 1. If $f \in C(V) \cap C R\left(V^{\circ}\right) \quad\left(E_{j}\right.$ 's are rectifiable, $\left.|f|_{E}<+\infty\right)$, then the formula

$$
\tilde{f}(z)=\lim _{m \rightarrow \infty} K_{m}(f ; z), \quad z \in H=\operatorname{int} W
$$

defines a holomorphic function $\tilde{f}$ in the domain $H$.

The same assertion is true under the condition of Theorem 2. We note the essential difference between the formulas (3) and (4). Carleman's formula (3) reconstructs a holomorphic function $f$ in the domain $D$ by its boundary values on the part $E$ of $b D$. The formula (4) defines holomorphic continuation of the function $f(t), t \in E$, in the domain $H$ if it is known that the function admits $C R$-continuation on $V^{\circ}$ - on the parts $V_{j}^{\circ}, j=1, \ldots, n$, of the boundary $b H$ with minimal complex structure (a single complex direction - variable $z_{j}$ - on each of these parts).

Using the formulas of the type (4) one can define $\tilde{f}$ on all parts of $b H$ with at least two complex directions. On $V_{j}^{\circ}$ 's the corresponding formulas reconstruct $f$ (for $f \in C(\mathscr{C}) \cap C R\left(V^{\circ}\right)$, Carleman's one-dimensional formula works on these parts of $b H)$. We put $\tilde{f}=f$ on $V$.

A comparison of these formulas provides the proof of the continuity of $\tilde{f}$ on $W^{\circ}$; using this fact one can prove the equation $|\tilde{f}|_{W^{\circ}}=|f|_{V^{\circ}}$. Finally, the continuity of $\tilde{f}(z), z \in W$, at the points $t \in E$ can be proved with the help of the following analogue of Nevanlinna's "two-constant theorem".

Lemma 2. Let $f \in C R\left(W^{\circ}\right),|f|_{W^{\circ}}=M<+\infty$, and for some fixed $j \in\{1, \ldots, n\}$ and any $t \in E$

$$
\limsup _{z \rightarrow t}\left|f_{j}(z)\right| \leqq m, \quad f_{j}=f \mid V_{j}^{0} .
$$

Then the following estimate holds:

$$
|f(z)| \leqq m^{1-h(z)} M^{h(z)}, \quad z \in W^{0} .
$$

\section{References}

[1] Beurling, A.: Analytic continuation across a linear boundary. - Acta Math. 128, 1972, 153-182.

[2] Epstein, H.: Generalization of the "edge of the wedge" theorem. - J. Math. Phys. 1, 1960, $524-531$.

[3] Epstein, H.: Some analytic properties of scattering amplitudes in quantum field theory. Particle symmetiies and axiomatic field theory. Vol. 1: Axiomatic field theory. Brandeis University Summer Institute in Theoretical Physics, 1965. Gordon and Breach, New York-London-Paris, 1966, 1-133.

[4] Martineau, A.: Le "edge of the wedge theorem" en théorie des hyperfonctions de Sato. - Proc. International Conference on Functional Analysis and Related Topics. Tokyo, 1969. Univ. of Tokyo Press, Tokyo, 1970, 95-106. 
[5] Rudin, W.: Lectures on the edge-of-the-wedge theorem. - Regional Conference Series in Mathematics 6. American Mathematical Society, Providence, R. I., 1971.

[6] SICIAK, J.: Separately analytic functions and envelopes of holomorphy of some lower dimensional subsets of $C^{n}$. - Ann. Polon. Math. 22, 1969/70, 145-171.

[7] Vladimirov, V. S.: Methods in the theory of functions of several complex variables. - Izdat. "Nauka", Moscow, 1964 (Russian).

[8] Vladimirov, V. S.: Bogoliubov's "edge of the wedge" theorem, its development and applications. - Problems of theoretical physics. Izdat. "Nauka", Moscow, 1969, 61-67 (Russian).

[9] Vladimirov, V. S. : Functions of several complex variables in mathematical physics. - Problems of mathematics and mechanics. Izdat. "Nauka", Novosibirsk, 1983, 15-32 (Russian).

\author{
Academy of Sciences of USSR \\ Steklov Mathematical Institute \\ ul. Vavilova, 42 \\ 117966 Moscow GSP-1 \\ USSR
}

Received 3 July 1984 